\title{
Video-assisted thoracoscopic thymectomy: bilateral approach
}

\author{
Jury Brandolini'1,2 \\ 'Department of Thoracic Surgery, Maggiore Teaching Hospital, Bologna 40133, Italy. \\ ${ }^{2}$ Department of Thoracic Surgery, Sant'Orsola University Hospital, Bologna 40138, Italy.
}

Correspondence to: Dr. Jury Brandolini, Department of Thoracic Surgery, Maggiore Teaching Hospital, Bologna 40133, Italy; Department of Thoracic Surgery, Sant'Orsola University Hospital, Bologna 40138, Italy.

E-mails: jury.brandolini@ausl.bologna.it; jury.brandolini@gmail.com

How to cite this article: Brandolini J. Video-assisted thoracoscopic thymectomy: bilateral approach. Mini-invasive Surg 2020;4:45. http://dx.doi.org/10.20517/2574-1225.2020.27

Received: 27 Feb 2020 First Decision: 27 Apr 2020 Revised: 13 May 2020 Accepted: 21 May 2020 Published: 19 Jul 2020

Academic Editors: Noriyoshi Sawabata, Piergiorgio Solli Copy Editor: Cai-Hong Wang Production Editor: Jing Yu

\begin{abstract}
Aim: The advantages and feasibility of video-assisted thoracoscopic surgery (VATS) in the surgical management of early resectable thymoma and thymic hyperplasia have largely been described and adopted in many thoracic surgery units. In order to allow for resection of all immunogenic thymic cells in patients with myasthenia gravis, surgical removal of the whole thymus gland including perithymic and pericardiophrenic fatty tissue becomes imperative. It is also important to achieve radical resection and excision in cases of thymoma.
\end{abstract}

Methods: Numerous technical variations of VATS thymectomy have been reported in literature. In this study, the surgical technique of a minimally invasive, extended thymectomy through a bilateral approach is illustrated with key features highlighted.

Results: In our experience, no conversion to the open transternal approach, surgical mortality or major complications were observed; the median length of hospital stay was 3 days.

Conclusion: Bilateral video-assisted extended thymectomy is an effective, safe and well-tolerated approach, with surgical benefits and clinical outcomes similar to other thoracoscopic techniques.

Keywords: Thymoma, video-assisted thoracic technique, thymectomy, bilateral video-assisted thoracoscopic surgery

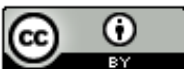

(C) The Author(s) 2020. Open Access This article is licensed under a Creative Commons Attribution 4.0 International License (https://creativecommons.org/licenses/by/4.0/), which permits unrestricted use sharing, adaptation, distribution and reproduction in any medium or format, for any purpose, even commercially, as long as you give appropriate credit to the original author(s) and the source, provide a link to the Creative Commons license, and indicate if changes were made.

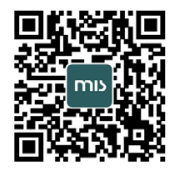




\section{INTRODUCTION}

Since the origin of this procedure, conventional open thymectomy has been considered the gold standard for the treatment of patients with thymomatous masses ${ }^{[1]}$. A gradual transition to minimally invasive techniques though, has become evident within the surgical community. In 1993, Coosemans et al. ${ }^{[2]}$ reported the first cases of video-assisted thoracoscopic surgery (VATS) thymectomy as a safe and effective approach, with or without additional trans-cervical incision.

With the improvement of technology, however, in terms of optical and surgical instrumentation, VATS thymectomy has become increasingly popular. Compared to the standard open technique, minimallyinvasive thymectomy has the advantages of reducing surgical trauma, less intraoperative blood loss and duration of postoperative pleural drainage, less postoperative pain, reduced hospital length of stay, better aesthetic result, rapid recovery of lung function and lower complications ${ }^{[3]}$.

Many retrospectives studies comparing open trans-sternal thymectomy to VATS thymectomy have reported no significant difference in terms of adverse events, surgical extent, rate of Ro resection, peri- and post-operative complications. Otherwise, faster recovery times were demonstrated in patients treated with bilateral thoracoscopic thymectomy for patients with $\mathrm{MG}^{[4]}$.

Worldwide, VATS is now used in the surgical treatment of early resectable thymomas and thymic hyperplasia in many thoracic surgery units. In the literature, numerous technical variations to VATS thymectomy have been described and the final choice depends on the individual surgeon's preferences and expertise. A bilateral approach may achieve a more radical thymectomy, as described by some surgeons, either alone or together with an additional cervical or sub-xyphoid incision ${ }^{[4-8]}$.

The choice of the first side of the thorax to be approached varies according to the surgeon's experience and preference. The intraoperative steps may also vary: some surgeons prefer to start dissection from the right side and divide the thymic veins from the left, while others approach the thymic veins from the left first, and some authors start dissecting the thymic veins from the right ${ }^{[6,9,10]}$.

In this study, the surgical technique of a minimally invasive, extended thymectomy through a bilateral approach is illustrated with key features highlighted.

\section{METHODS}

All operations were carried out under general anaesthesia with double-lumen intubation. The patients were placed in a semi-supine decubitus position with the hemithorax raised to about $30^{\circ}$ from the horizontal plane, and widely prepped to allow simple exposure of both sides. The entire chest is elevated from the table by a soft gel roll placed under the spine with both arms extended overhead for wide exposure of the two hemithoraces [Figure 1]. The head is also flexed, in order to move the thymus inferiorly into the mediastinum, out of the cervical neck. The assistant stands beside the operating surgeon, while the scrub is placed facing the operating surgeon.

\section{Step 1: right side}

The right side is accessed first. With the surgical table tilted slightly towards the left, an initial 10-mm trocar is inserted through the 5th intercostal space (ICS) along the anterior-axillary line; two additional 5-mm ports are then placed in the fifth ICS along the mid-clavicular line, and in the third ICS along the midaxillary line respectively [Figure 1]. CO2 insufflation is used during the whole procedure (the pressure is commonly maintained al $6 \mathrm{mmHg}$, and flow around $6 \mathrm{~mL} / \mathrm{min}$ ), in order to favour right lung collapse and facilitate dissection. A 30 -degree $(5 \mathrm{~mm}$ or $10 \mathrm{~mm})$ scope is used to allow visualisation of the mediastinal structures from multiple perspectives. 

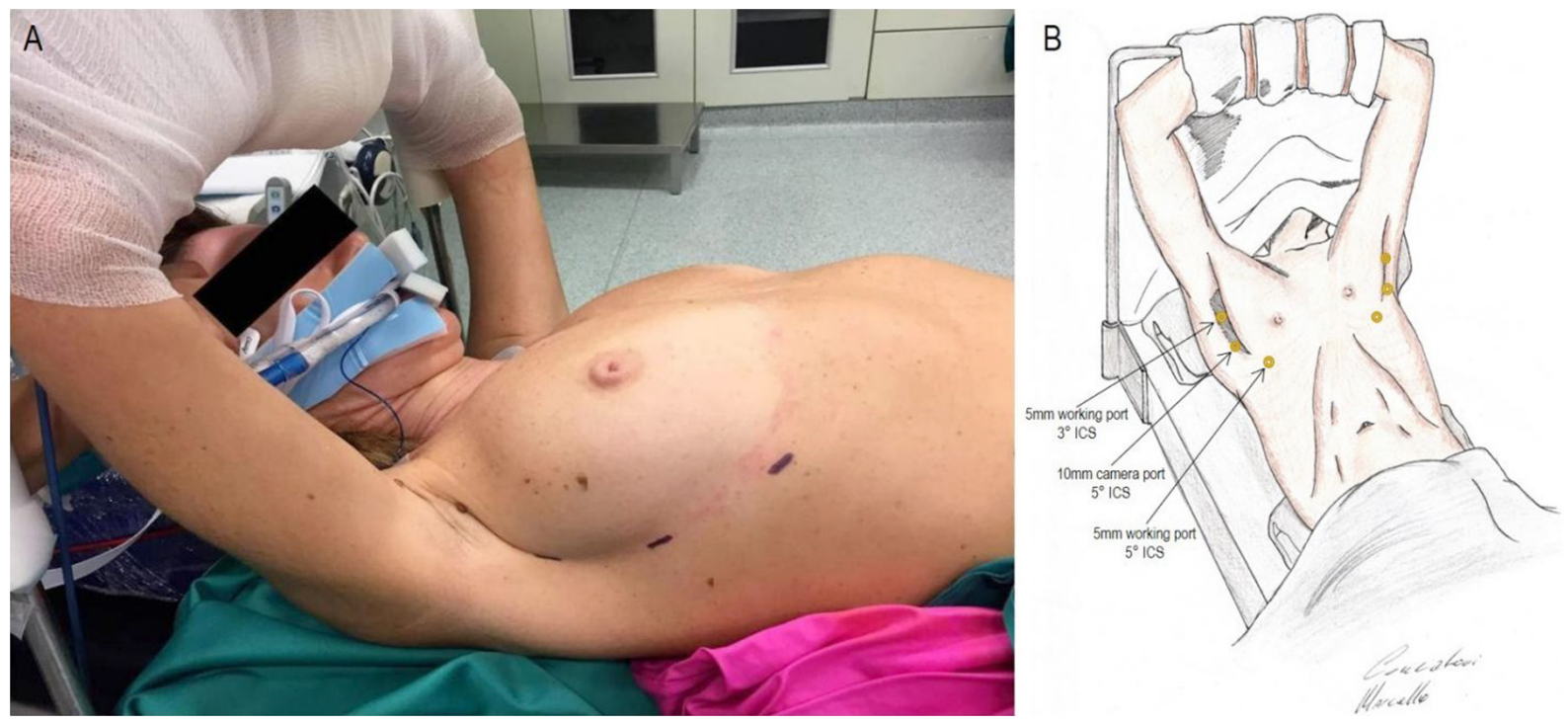

Figure 1. Young patient positioned for bilateral video-assisted thoracoscopic surgery (VATS) thymectomy; right-sided approach. The entire chest is lifted from the table by a soft gel shoulder roll with both arms extended overhead to allow full exposure of the bilateral hemithoraces (A); port placement for bilateral VATS thymectomy (B). ICS: intercostal space

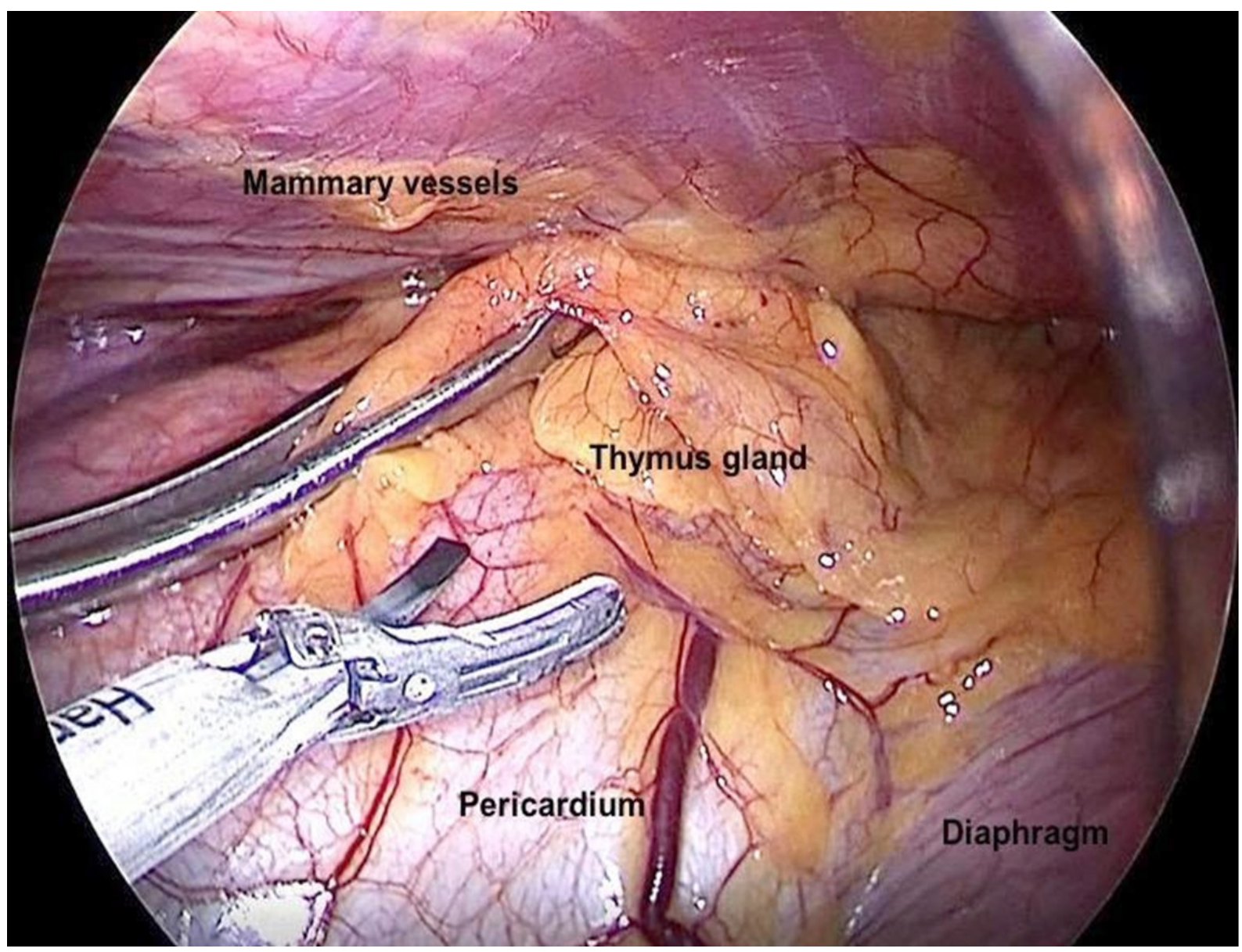

Figure 2. Right-sided view: the dissection starts inferiorly by mobilizing the thymus from the pericardium 


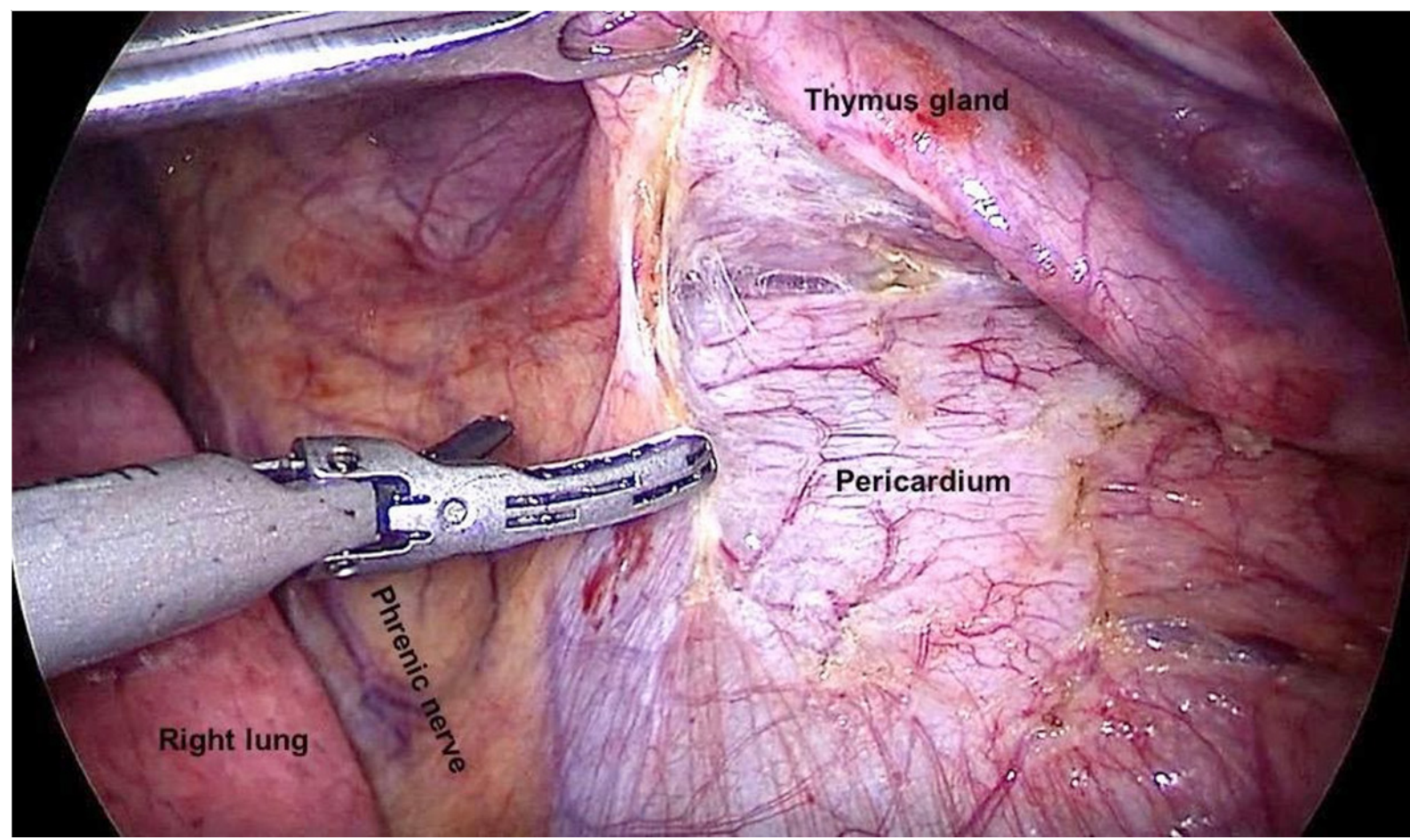

Figure 3. Right-sided view: the thymic dissection is performed cranially along the phrenic nerve with a harmonic scalpel. The main anatomic landmarks are indicated

After inspection of the mediastinal and pleural surfaces to confirm the absence of metastases, the dissection begins by grasping the fat pad in the right cardiophrenic angle [Figure 2]; the mediastinal pleura is now opened anterior to the right phrenic nerve with a harmonic scalpel (Ultracision Harmonic; Johnson \& Johnson, NJ) [Figure 3]. The right lobe of the thymus gland and surrounding fat are mobilized from the diaphragm inferiorly, the pericardial surface medially, and from the posterior aspect of the sternum [Figure 4]. The dissection is carried superiorly, in a caudocranial direction, along the right phrenic nerve in order to identify the right internal mammary vein and to reach the confluence of the superior vena cava and the left innominate vein [Figure 5]. Care must be taken to prevent stretch and thermal injury to the nerve and mediastinal vessels.

Dissection is achieved by combining blunt and sharp dissection. The left innominate vein is clearly identified and the right cervical horn can be grasped and gradually pulled down into the pleural cavity, freeing it up from the surrounding fat tissue, in order to achieve complete mobilization with identification of the thymo-thyroid ligaments, which are then divided. The left horn is visualised and dissected with the same technique from the right hemithorax.

The body of the thymus gland is mobilized downwards in order to expose the thymic veins (veins of Keynes), which are dissected and divided along the innominate vein, using an endoscopic clip applier (click a V, GrenaR or titanium clips) or with an energy device. All arterial communications with the internal mammary arteries are divided; after mobilisation of both the superior poles, dissection can now be continued towards the left chest.

After completion of the right-sided dissection, the retrosternal space with the left parietal pleura is widely opened, and the specimen is then pushed into the left pleural cavity. 


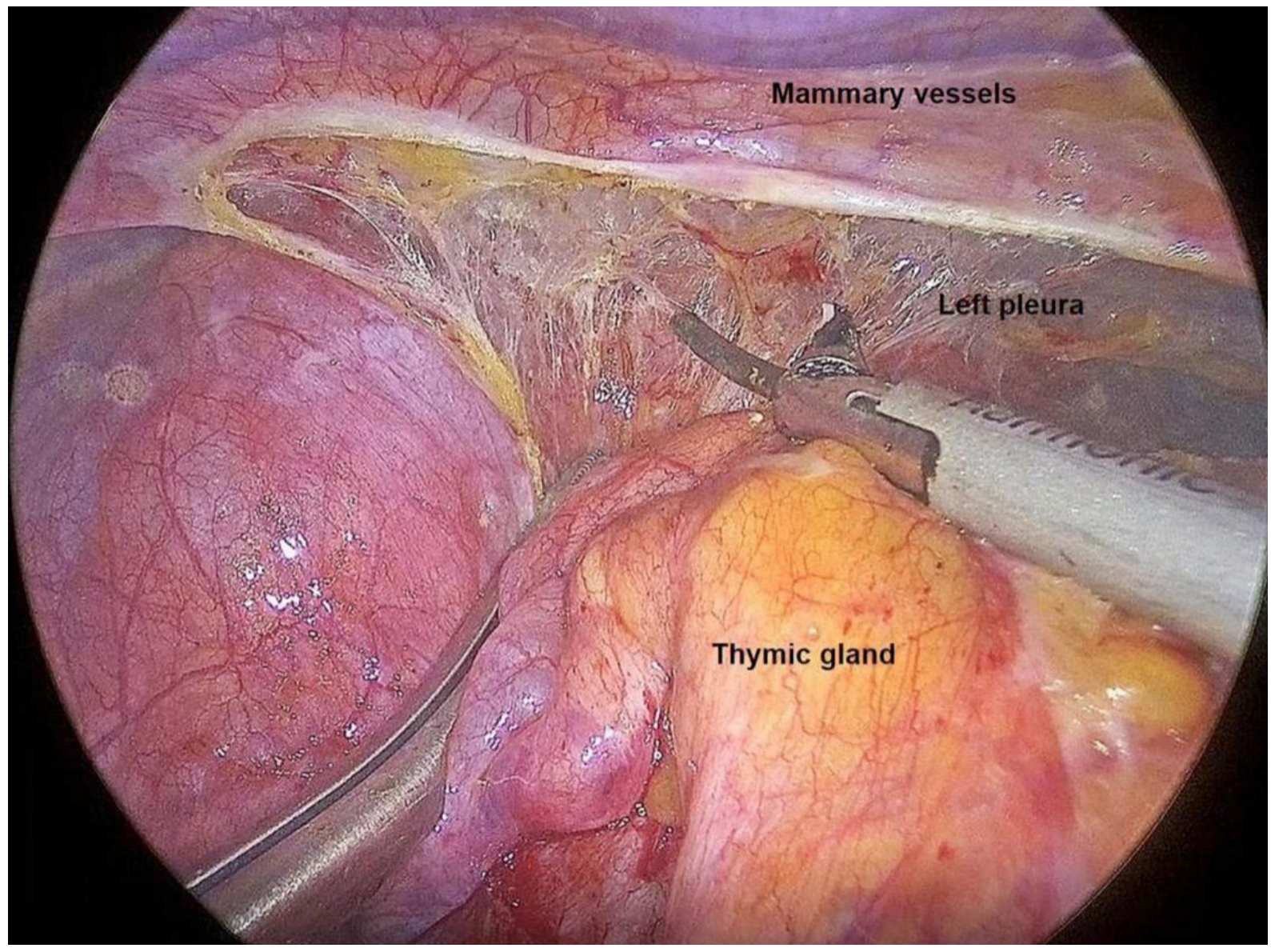

Figure 4. Right-sided video-assisted thoracoscopic surgery view: the right lobe of the thymus and surrounding fat are mobilized from the posterior aspect of the sternum. The anatomic landmarks are indicated

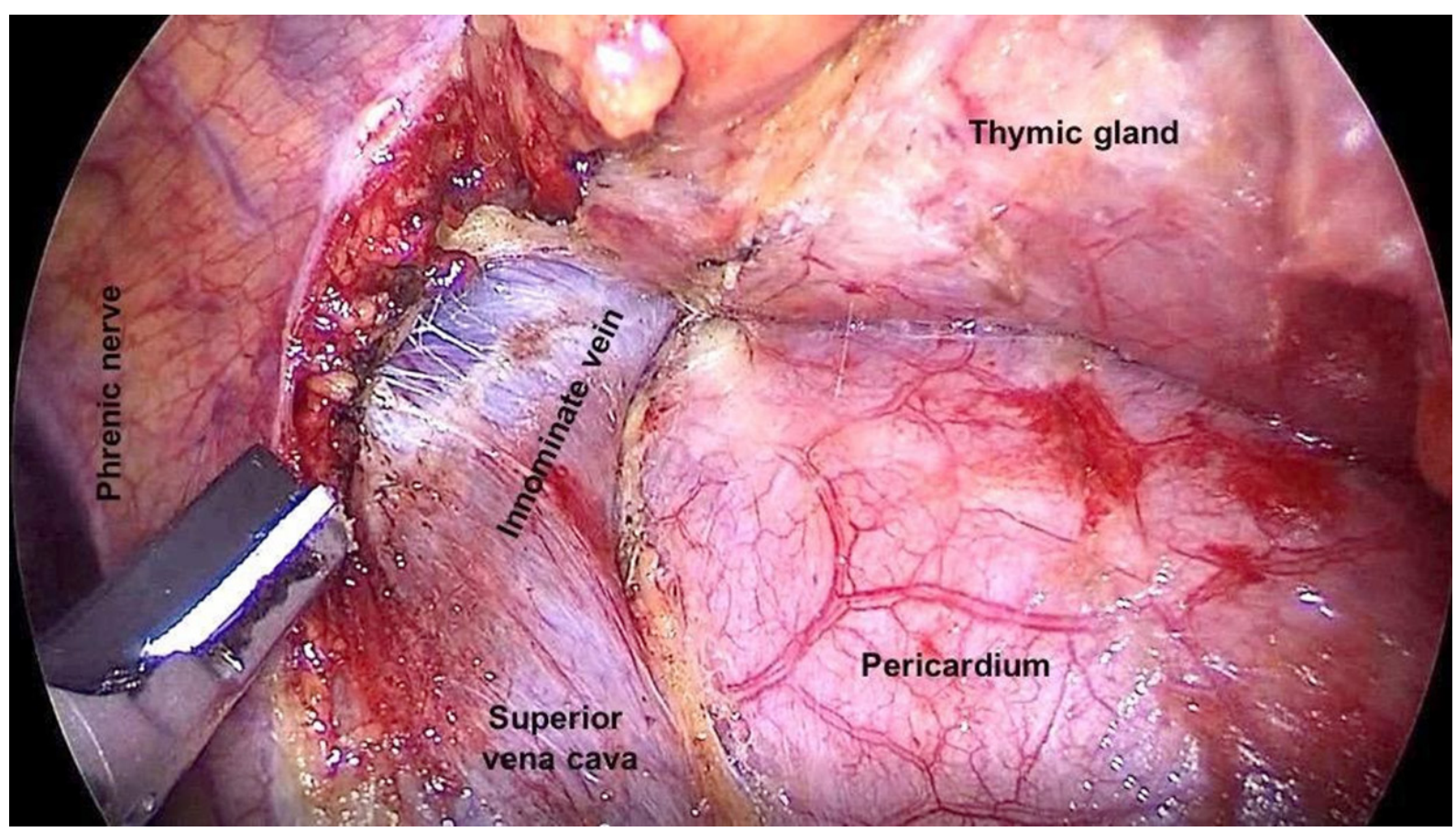

Figure 5. Right-sided video-assisted thoracoscopic surgery view: dissection at the level where the left innominate vein joins the superior vena cava. The anatomic landmarks are indicated 


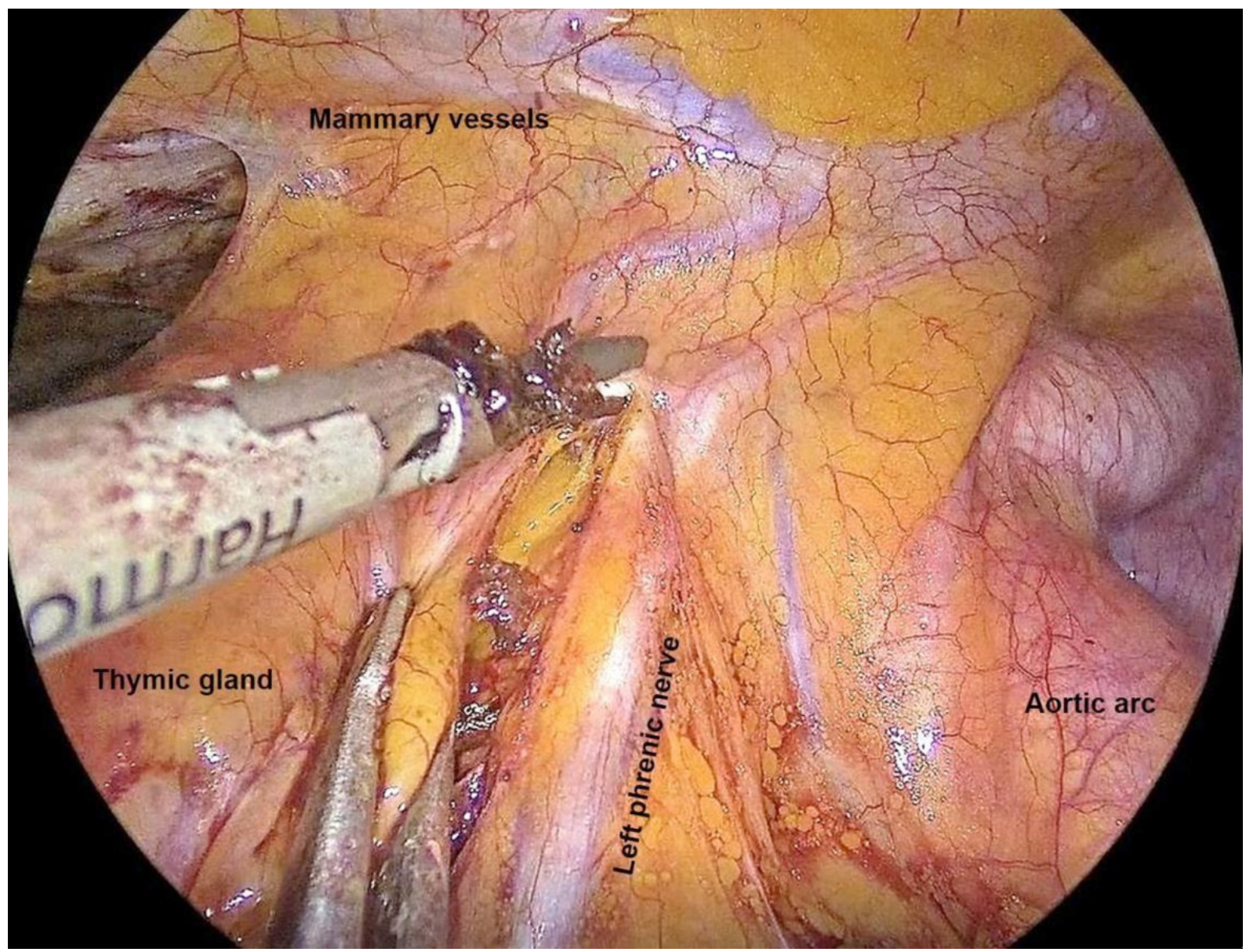

Figure 6. Left-sided video-assisted thoracoscopic surgery view. The thymic gland is freed from the left phrenic nerve with a harmonic scalpel (Ultracision Harmonic; Johnson \& Johnson, New Brunswick, NJ) and the dissection continues cephalad in order to access the thoracic inlet and the previously dissected right side of the procedure

\section{Step 2: left side}

Right lung ventilation is re-initiated. The high-definition camera is inserted through a 5- or 10-mm port, placed in the 5th ICS along the mid-axillary line within the inframammary fold. Two additional ports are then inserted under direct visualisation; a $5-\mathrm{mm}$ port is inserted through the $3 \mathrm{rd}$ ICS along the anterior axillary line and another 5-mm port is placed in the 5th ICS along the mid-clavicular line.

Next, the dissection starts at the level of the left cardiophrenic angle, by grasping the thymus and the fat pad anterior to the left phrenic nerve. The thymus gland is mobilized by dissecting from the pericardial layer. When the retrosternal plane is opened widely, the dissection continues cranially along the left phrenic nerve, in order to join the thoracic inlet superiorly, and the previous site of dissection on the posterior aspect of the sternum [Figure 6].

All thymic tissue and the anterior mediastinal fat are now completely mobilized. Removal of the specimen is finally performed using an Endobag after enlarging the 10-mm port site. Usually, at the end of the procedure, a 24-F chest tube is placed in both hemithorces through the lowest existing port incision for postoperative drainage of fluid and air. Correct lung re-inflation is then directly visualized and all port sites are closed with absorbable sutures (Figure 7 - final right-side view). 


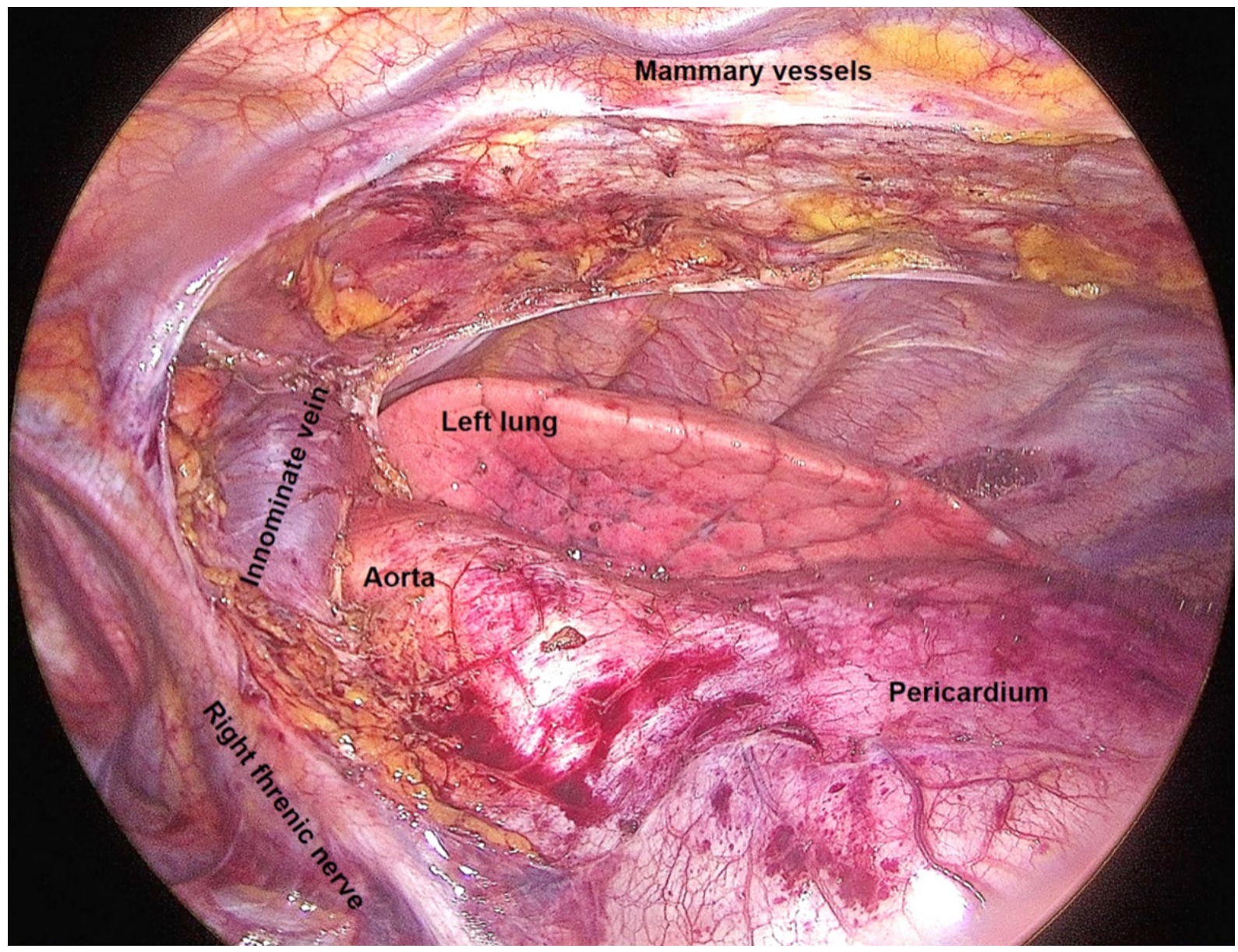

Figure 7. Final view from the right; the anatomic landmarks are indicated

\section{Tips and tricks}

(1) We suggest performing VATS thymectomy using double-lumen intubation, which may be useful in case of lung infiltration from a thymoma.

(2) In order to avoid the risk of capsular break and spillage of thymic tissue, we recommend avoiding grasping of the thymus gland directly, i.e., a "touch-free" technique is preferable.

(3) It is helpful to perform all phases of the surgery and the entire dissection of the mediastinal fat with energy-based tissue sealing devices such as the harmonic scalpel (Ultracision Harmonic; Johnson \& Johnson, NJ) or electrothermal bipolar tissue sealing system (LigaSure, Valleylab Inc., USA), both of which can also be used for dividing small thymic vessels.

(4) During minimally invasive thymectomy, $\mathrm{CO} 2$ insufflation in the chest cavity offers various advantages: faster lung deflation; increased operative space by pushing the mediastinum and diaphragm towards the opposite chest cavity and away from the operative area; continuous circulation of cautery smoke and aerosolized ultrasonic vapor during dissection to allow better visibility of the surgical field; and it helps in dissecting and visualizing tissue planes.

(5) Bilateral VATS thymectomy offers the possibility to perform the whole thymic resection from either the right or left side, in the event of pleural adhesion on one side as both phrenic nerves are clearly visualized.

In our experience, no conversion to the open transternal approach, surgical mortality or major complications were observed. The median operative time and blood loss were $150 \mathrm{~min}(+/-20 \mathrm{~min})$ and $20 \mathrm{~mL}$ $(+/-20 \mathrm{~mL})$ respectively and the median length of hospital stay was 3 days. 


\section{DISCUSSION}

The aim of thymectomy in patients with myasthenia gravis (MG) is the complete removal of the thymus and perithymic tissue to eradicate all immunogenic thymic cells and potentially viable thymic tissue in patients with MG to minimize disease persistence or increasing relapse rates. In cases of malignancy when a thymoma is diagnosed, it is still crucial to achieve radical en bloc excision of the residual thymic gland to improve both overall survival and the risk of local recurrence.

With a left-sided approach for thymectomy, dissection of fat tissue in the right cardio-phrenic angle and within the confluence of the superior vena cava and innominate vein can be difficult, while the resection of the thymus gland with fat tissue in the left cardio-phrenic angle and at the level of the aorto-pulmonary window may be limited with a right-sided approach. On the contrary, the bilateral view improves the approach to the left innominate vein and offers a clear and close view of both phrenic nerves. Moreover, the bilateral view might be especially helpful in thymomas.

An additional cervical incision within the neck may be helpful for a more extended excision of all residual thymus at the level of the upper cervical poles but in our experience, the bilateral VATS technique can safely achieve radical dissection of both superior thymic horns. Some sort of bilateral view, especially of the controlateral phrenic nerve, might be achieved by adding a sub-xyphoid port to the unilateral approach (see below).

We believe that correct positioning of the trocars and the patient's position are crucial to facilitate surgical dissection, avoid instrumental conflicts within the thorax and to reduce operating time. We routinely use an energy-based tissue sealing device during the whole procedure (ultrasound or radiofrequency are equivalent depending on personal experience and preference) for tissue handling, dissection and sealing vessels (Keynes veins).

Among the various minimally invasive approaches, the subxiphoid thymectomy described by Kido et al. ${ }^{[1]}$ in 1999 is gaining interest and popularity among thoracic surgeons. The two main advantages of this approach are reduction of postoperative pain and cosmetic results because of the small incisions ( 1 or 2 ports for access) and the possibility to avoid intercostal nerve damage. As shown by Suda et al. ${ }^{[12]}$, in comparison with a lateral VATS approach, the subxiphoid thymectomy is associated with reduced consumption of postoperative analgesics and perioperative blood loss. A similar operative time was observed in the two groups ${ }^{[12]}$. This technique seems to be able to overcome some technical difficulties of the VATS operation, such as the small working space, different viewpoint from a median sternotomy and bilateral phrenic nerve control. On the other hand, the subxiphoid approach is not widely used because of its unfamiliarity among thoracic surgeons and difficult intraoperative control of bleeding in the event of major vessel injury such as bleeding of the left innominate vein.

The definition and indications for VATS thymectomy in the treatment of early-stage thymomas are summarized in the ITMIG reccomandations ${ }^{[13]}$. Some authors disagree with a minimally invasive approach for large tumours because the dissection might be difficult and tumor manipulation might translate into intraoperative seeding of the pleural space, which would compromise the procedure ${ }^{[14]}$.

Nevertheless, some earlier studies comparing robotic-assisted thymectomy with trans-sternal thymectomy showed that large thymic tumors can be managed by the robotic approach, which has improved threedimensional visualization, increased freedom of instrument motion for precise dissection and permits radical dissections, even of thymomas $>4 \mathrm{~cm}$ in diameter, while reducing the risk of capsular injury and providing all the benefits in postoperative recovery of the minimally-invasive approach to the patient ${ }^{[15]}$. 


\section{DECLARATIONS}

\section{Authors' contributions}

The author contributed solely to the article.

\section{Availability of data and materials}

Not applicable.

\section{Financial support and sponsorship}

None.

\section{Conflicts of interest}

The author declared that there are no conflicts of interest.

\section{Ethical approval and consent to participate}

Not applicable.

\section{Consent for publication}

Written informed consent was obtained from the patients for publication of the manuscript and associated images.

\section{Copyright}

(C) The Author(s) 2020.

\section{REFERENCES}

1. Okumura M, Ohta M, Tateyama H, Nakagawa K, Matsumura A, et al. The World Health Organization histologic classification system reflects the oncologic behavior of thymoma: a clinical study of 273 patients. Cancer 2010;94:624-32.

2. Coosemans W, Lerut TE, Van Raemdonck DE. Thoracoscopic surgery: the Belgian experience. Ann Thorac Surg 1993;56:721-30.

3. Rückert JC, Gellert K, Müller JM. Operative technique for thoracoscopic thymectomy. Surg Endosc 1999;13:943-6.

4. Lee CY, Kim DJ, Lee JG, Park IK, Bae MK, et al. Bilateral video-assisted thoracoscopic thymectomy as a surgical extended thymectomy with more favorable early surgical outcomes for myasthenia gravis patients. Surg Endosc 2011;25:849-54 .

5. Bromberger B, Sonett J. Bilateral VATS thymectomy in the treatment if myasthenia gravis. Video Assist Thorac Surg 2017;2:12.

6. Chang PC, Chou SH, Kao EL, Cheng YJ, Chang HY, et al. Bilateral video-assisted thoracoscopic thymectomy vs. extended transsternal thymectomy in myasthenia gravis: a prospective study. Eur Surg Res 2005;37:199-203.

7. Shigemura N, Shiono H, Inoue N, Minami M, Ohta M, et al. Inclusion of the transcervical approach in video-assisted thoracoscopic extended thymectomy (VATET) for myasthenia gravis: a prospective trial. Surg Endosc 2006;20:1614-8.

8. Lee CY, Kim DJ, Lee JG, Park IK, Bae MK, et al. Bilateral video-assisted thoracoscopic thymectomy has a surgical extent similar to that of transsternal extended thymectomy with more favorable early surgical outcomes for myasthenia gravis patients. Surg Endosc $2011 ; 25: 849-54$.

9. Siwachat S, Tantraworasin A, Lapisaterpun W, Ruengorn C, Taioli E, et al. Comparative clinical outcomes after thymectomy for myasthenia gravis: thoracoscopic versus transternal approach. Asian J Surg 2018;41:77-85.

10. Gung Y, Zhang H, Li S, Wang Y. Sternotomy versus video-assisted thoracoscopic surgery for thymectomy of myasthenia gravis patients: a meta-analysis. Asian J Endosc Surg 2016;9:285-94.

11. Kido T, Hazama K, Inoue Y, Tanaka Y, Takao T. Resection of anterior mediastinal masses through an infrasternal approach. Ann Thorac Surg 1999;67:263-5.

12. Suda T, Hachimaru A, Tochii D, Maeda R, Tochii S, et al. Video-assisted thoracoscopic thymectomy versus subxiphoid single-port thymectomy: initial results. Eur J Cardiothorac Surg 2016;49:i54-8.

13. Toker A, Sonett J, Zielinski M, Rea F, Tomulescu V, et al. Standard terms, definitions, and policies for minimally invasive resection of thymoma. J Thorac Oncol 2011;6:S1739-42.

14. Girard N, Mornex F, Van Houtte P, Cordier JF, van Shil P. Thymoma: a focus on current therapeutic management. J Thorac Oncol 2009;4:119-26.

15. Kneuertz J, Kamel MK, Stiles BM, Lee BE, Rahouma M, et al. Robotic thymectomy is feasible for large thymomas: a propensitymatched comparison. Ann Thorac Surg 2017;104:1673-8. 